Commun. Korean Math. Soc. 29 (2014), No. 3, pp. 463-478

http://dx.doi.org/10.4134/CKMS.2014.29.3.463

\title{
CRITERIA FOR A SYMMETRIZED MONOMIAL IN B(3) TO BE NON-HIT
}

\author{
Ali S. JANFADA
}

Dedicated to Reg M. W. Wood on the occasion of his 75th birthday

\begin{abstract}
We find criteria for symmetrized monomials to be non-hit in the $\mathcal{A}_{2}$-algebra of symmetric polynomials in three variables, where $\mathcal{A}_{2}$ is the mod 2 Steenrod algebra.
\end{abstract}

\section{Introduction}

Consider the polynomial algebra $\mathrm{P}(n)=\mathrm{F}_{2}\left[x_{1}, \ldots, x_{n}\right]=\bigoplus_{d \geq 0} \mathrm{P}^{d}(n)$ viewed as a graded left module over the Steenrod algebra $\mathcal{A}_{2}$. Denote by $\mathrm{B}(n)=\mathrm{P}(n)^{\Sigma_{n}}$ the $\mathcal{A}_{2}$-submodule of $\mathrm{P}(n)$ consisting of the symmetric polynomials, where $\Sigma_{n}$ denotes the symmetric group on $n$ letters acting on the right of $\mathrm{P}(n)$ by matrix substitution [9].

The algebra $\mathrm{P}(n)$ and its subalgebra $\mathrm{B}(n)$ realize, respectively, the cohomology of the product of $n$ copies of infinite real projective space and the cohomology of the classifying space $B O(n)$ of the orthogonal group $O(n)$. The ideal $\mathrm{L}(n)$ in $\mathrm{P}(n)$ generated by $\sigma_{n}=x_{1} \cdots x_{n}$ can be identified with the cohomology of the $n$-fold smash product of infinite real projective space in positive dimensions.

A homogeneous element $f$ of grading $d$ in a graded left $\mathcal{A}$-module $\mathrm{M}$ is hit if there is a finite sum $f=\sum_{i>0} S q^{i}\left(h_{i}\right)$, called a hit equation, where the preimages $h_{i} \in \mathrm{M}$ have grading strictly less than $d$. We define $f$ and $g$ to be equivalent, write $f \cong g$, whenever $f-g$ is hit. The hit problem is to discover criteria for elements of $\mathrm{M}$ to be hit and find minimal generating sets for $\mathrm{M}$ as an $\mathcal{A}$-module.

We call the hit problem for $\mathrm{B}(n)$ the symmetric hit problem. In particular, a hit equation in $\mathrm{B}(n)$, called a symmetric hit equation, is the finite $\operatorname{sum} f=$ $\sum_{i>0} S q^{i}\left(h_{i}\right)$, where now $f$ and the pre-images $h_{i}$ are symmetric polynomials. In this case we say that $f$ is symmetrically hit. The symmetric hit problem at

Received January 30, 2014; Revised June 14, 2014.

2010 Mathematics Subject Classification. 55S10.

Key words and phrases. hit problem, symmetric hit problem, Steenrod algebra. 
the prime 2 has been studied by the author and Reg M. W. Wood $[2,5,6]$ and quotients had earlier been studied by Frank Peterson [8].

We shall adopt the notation and terminology of $[2,3]$. In particular, we use the convenient binary block [1] as a geometric device for keeping track of the action of Steenrod squares on monomials. A monomial $f=x_{1}^{d_{1}} \cdots x_{n}^{d_{n}}$ has an associated matrix whose row entries are the digits, in reversed binary expansion, of the exponents $d_{i}$. We shall adopt the convention of denoting a monomial by a lower case letter and its binary block by the corresponding upper case letter. For more details about blocks see $[2,6]$.

Many results and processes concerning the action of the Steenrod squares are easier to understand when expressed in the language of blocks and we shall regard a monomial $f$ and its block $F$ as interchangeable. An example is the $\omega$-vector $\omega(f)$ of a monomial $f$, which is the vector $\omega(F)$ of column sums of $F$ [5]. The row sums of $F$, which is the $\omega$-vector of the transpose of $F$, form the $\alpha$-vector of $F$, denoted by $\alpha(F)$. The components of the $\alpha$-vector are arranged in increasing order to form the $\alpha^{*}$-vector.

By ordering vectors lexicographically, we obtain the partial orders on the set of monomials in $\mathrm{P}(n)$, the $\omega$-order, the $\alpha$-order, and the $\alpha^{*}$-order. The $\omega$-order will be dominant throughout this work and when, say, we write $f$ is lower than $g$, we mean lower in the $\omega$-order.

By a spike we mean a monomial $x_{1}^{d_{1}} \cdots x_{n}^{d_{n}}$, where all exponents $d_{i}$ are in the form $2^{\lambda_{i}}-1$ for some non-negative integer $\lambda_{i}$. A minimal spike is a spike of the same degree with least $\omega$-vector. Throughout this paper we will denote minimal spikes by the symbol $\mathfrak{m}$.

The symmetrization of a monomial $f$ is the "smallest" symmetric polynomial $\sigma(f) \in \mathrm{B}(n)$ containing $f$ as a term. To be precise, $\sigma(f)=\sum_{i=1}^{t} f \pi_{i}$, where $\pi_{1}, \ldots, \pi_{t}$ run through a set of left coset representatives for the stabilizer of the monomial $f$ in $\Sigma_{n}$.

A basic part of the hit problem is to detect all the hit elements. In [3] the author exhibited a criterion for a monomial in $\mathrm{P}(3)$ to be hit. A similar tool in the symmetric hit problem is given in the present paper. In the 1variable case, the solution to the symmetric hit problem is the same as the hit problem since $\mathrm{B}(1)=\mathrm{P}(1)$ and the only non-hit monomials in $\mathrm{P}(1)$ are the spikes $x^{2^{s}-1}$. The situation for $n=2$ is similar as the only non-hit elements are the symmetrizations of spikes $[6]$.

The main criteria in the 3 -variable case are the following theorems, for the first two of which we have $\mu(d)=2$, while Theorem 1.3 is in the case $\mu(d)=1$. Here $\mu(d)$, for a positive integer $d$, stands for the smallest value of $k$ for which it is possible to write $d=\sum_{i=1}^{k}\left(2^{\lambda_{i}}-1\right)$, where $\lambda_{i}>0$. In Section 2 it is explained that why we do not need to $\mu \geq 3$ cases.

Theorem 1.1. Let $d=2^{t+s}+2^{t}-2$, where $t>0, s=0,1$. Assume $F$ is a block in $\mathrm{L}^{d}(3)$. Then $\sigma(F)$ is hit in $\mathrm{B}(3)$. 
In the following main theorem we use the standard splitting $F=F^{u} F^{t} F^{s}$ of a block $F$ which will be defined in Section 2 .

Theorem 1.2. Let $d=2^{t+s}+2^{t}-2$, where $t>0, s>1$. Assume $F$ is a non-spike block in $\mathrm{L}^{d}(3)$. Then $\sigma(F)$ is non-hit in $\mathrm{B}(3)$ if and only if $F$ satisfies either of the following two conditions.

(1) $\omega(F)=\omega(\mathfrak{M})$ and $F^{s}$ has exactly one zero row;

(2) F has one of the following forms up to permutation of rows.

$$
G_{1}^{\prime}=\begin{array}{llllll}
0 & - & 0 & 1 & - & 1 \\
1 & - & 1 \\
1 & - & 1
\end{array} \quad G_{2}^{\prime}=\begin{array}{llllllll}
1 & - & 1 & 1 & 1 & - & 1 \\
1 & & & 1 & 1 & 1 & - & 1 \\
0 & - & 0 & 1
\end{array}
$$

Theorem 1.3. Let $d=2^{s}-1, s>2$ and let $F$ be a non-spike block in $\mathrm{L}^{d}(3)$. Then $\sigma(F)$ is non-hit in $\mathrm{B}(3)$ if and only if either of the following two conditions is satisfied.

(1) At least two rows of $F$ are equal;

(2) $F$ has three distinct rows and $\omega(F)=(3,2, \ldots, 2)$.

Theorems 1.1, 1.2, and 1.3 are unpublished results from the author's thesis [2] stated in different point of views, without proof, in [6] as Theorems 3.6, 3.8, and 3.5, respectively. The aim of the present paper is to concentrate on the strategy of the proof, in particular, digital engineering Lemmas 3.1, 3.4, 3.6, and 3.7. The techniques used in these lemmas may be generalized for higher variables.

\section{Preliminary results}

Let $f$ be a monomial in $\mathrm{P}(n)$ and let $\pi_{1}, \ldots, \pi_{t}$ be left coset representatives for a subgroup of the stabilizer of $f$ in $\sum_{n}$. Then $\sum_{j=1}^{t} f \pi_{j}$ is symmetric but the expression may be zero. For example, the transfer $\tau(f)=\sum_{\pi \in \sum_{n}} f \pi$ of a monomial $f$ with two equal exponents is zero. Note that for any monomial $f$, $\tau(f)=\sigma(f)$ whenever $f$ has distinct exponents.

The general plan of action in tackling the hit problem in $\mathrm{P}(n)$ is to use the $\omega$-order as a potential function and try to manipulate monomials into equivalent polynomials of lower potential with the ultimate aim of achieving some kind of canonical forms. The idea is then to transfer these to $\mathrm{B}(n)$ by symmetrization. However, this is not always straightforward business [4, Example 1]. The following result [4, Thm. 3, Props. 3, 4] is an example of this strategy. Recall from group theory that an odd (resp. even) permutation is a product of an odd (resp. even) number of transpositions.

Proposition 2.1. Let $f$ be a monomial in $\mathrm{P}(n)$ with distinct exponents.

(1) An equivalence $f \cong g$ in $\mathrm{P}^{d}(n)$, for any polynomial $g$, symmetrizes to a symmetric equivalence $\sigma(f) \cong \tau(g)$ in $\mathrm{B}(n)$;

(2) If $f$ is hit, then $\sigma(f)$ is symmetrically hit; 
(3) If $f \cong f \pi^{\prime}$ in $\mathrm{P}(n)$ for some odd permutation $\pi^{\prime}$ in $\Sigma_{n}$, then $\sigma(f)$ is symmetrically hit.

The next result is a useful corollary of Proposition 2.1 in the 3-variable case. We denote by $\pi_{(i, j)}$ the element of $\Sigma_{n}$ switches $x_{i}$ and $x_{j}$ and leaves the other variables fixed.

Corollary 2.2. Let $f, g$ be monomials in $\mathrm{P}(3)$ such that $f$ has exactly two equal rows $i, j$ and $g$ has distinct rows. Suppose that $f \cong g+g \pi_{(i, j)}$ in $\mathrm{P}(3)$. Then $\sigma(f) \cong \sigma(g)$ in $\mathrm{B}(3)$.

The symmetric version of the Peterson conjecture [8] reduces the symmetric hit problem to the case where $\mu(d) \leq n$.

Theorem 2.3. $\operatorname{dim}\left(\mathrm{Q}^{d}(\mathrm{~B}(n))\right)=0$ if and only if $\mu(d)>n$.

The next result [5, Thm. 2.3], analogous to Kameko's theorem [7, Thm. 4.2 ], reduces the symmetric hit problem to a study of symmetric polynomials in degrees $d$ where $\mu(d)<n$.

Theorem 2.4. Let $d$ and $n$ satisfy $\mu(d)=n$. Let $\mathfrak{m}$ be a minimal spike in $\mathrm{P}^{d}(n)$ and $u$ be the largest integer for which $\mathfrak{b}=\left(x_{1} \cdots x_{n}\right)^{2^{u}-1}$ divides $\mathfrak{m}$. Let also $d^{\prime}=\left(d-n\left(2^{u}-1\right)\right) / 2^{u}$. Then the linear transformation of vector spaces $\mathrm{B}^{d^{\prime}}(n) \rightarrow \mathrm{B}^{d}(n)$, given by $f \mapsto \mathfrak{b} f^{2^{u}}$ for $f \in \mathrm{B}^{d^{\prime}}(n)$, induces a vector space isomorphism

$$
\phi: \mathrm{Q}^{d^{\prime}}(\mathrm{B}(n)) \rightarrow \mathrm{Q}^{d}(\mathrm{~B}(n)) .
$$

The symmetric hit problem can be even more reduced to a study of monomials not lower than a minimal spike [5, Thm. 2.2].

Theorem 2.5. Let $f$ be a monomial in $\mathrm{P}^{d}(n)$ where $\mu(d) \leq n$. If $f$ is lower in the $\omega$-order than a minimal spike in $\mathrm{P}^{d}(n)$, then $\sigma(f)$ is hit in $\mathrm{B}(n)$.

We recall from $[2,6]$, in the 3 -variable case, the standard (vertical) splitting of blocks into sub-blocks whose length match the lengths of the $i$-sections, $1 \leq i \leq 3$, of a minimal spike. Let $n=3$ and suppose $\mu(d) \leq 3$. Then we can write $d=2^{u+t+s}+2^{u+t}+2^{u}-3$, where $u, t, s$ are non-negative integers determined in the following way. The $\omega$-vector of a minimal spike $\mathfrak{M}$ in $\mathrm{P}^{d}(3)$ has the form

$$
\omega(\mathfrak{M})=(3, \ldots, 3,2, \ldots, 2,1, \ldots, 1),
$$

where the 3 -section has length $u$, the 2 -section has length $t$ and the 1 -section has length $s$. If any of $u, t, s$ are zero, then the corresponding section is empty. Any block $B$ in $\mathrm{P}^{d}(3)$, not below the minimal spike, has a standard splitting $B=B^{u} B^{t} B^{s}$, where $B^{u}$ has $u$ columns, $B^{t}$ has $t$ columns and $B^{s}$ has $s$ columns. For purely numerical reasons we have

$$
\omega\left(B^{u}\right)=\omega\left(\mathfrak{M}^{u}\right)=(3, \ldots, 3), \quad \omega\left(B^{t}\right)=\omega\left(\mathfrak{M}^{t}\right)=(2, \ldots, 2) .
$$

Furthermore, $\operatorname{deg}\left(B^{s}\right)=\operatorname{deg}\left(\mathfrak{M}^{s}\right)=2^{s}-1$ but $B^{s}$ may be higher than $\mathfrak{M}^{s}$ in the $\omega$-order and its last column may be zero. If $B$ is not hit, then by Theorem 
$2.5 B$ is not lower than the minimal spike $\mathfrak{M}$. We may restrict attention to this situation and the following constraint on $\omega$ orders [3, Lem. 3.4].

Lemma 2.6. Let $B$ be a block in $\mathrm{P}(3)$ of degree $d=2^{u+t+s}+2^{u+t}+2^{u}-3$, where $u, s, t \geq 0$ are as in the standard splitting above. Assume $B$ is not lower than the minimal spike in degree $d$. Then the standard splitting $B=B^{u} B^{t} B^{s}$ satisfies

$$
\omega\left(B^{u}\right)=(3, \ldots, 3), \quad \omega\left(B^{t}\right)=(2, \ldots, 2),
$$

and $B^{s}$ is a juxtaposition of blocks with $\omega$-vectors of types

$$
\omega^{\prime}=(1, \ldots, 1), \quad \text { or } \quad \omega^{\prime \prime}=(3,2, \ldots, 2,0),
$$

in any order, where in the case of $\omega^{\prime \prime}$, which exist only when $s>1$, the initial 3 and final 0 have to be present, except in the last block position, but the 2-section can be empty. In particular, if $B$ is non-hit, then the standard splitting of $B$ satisfies the above properties.

The proofs of the main Theorems 1.1, 1.2, and 1.3, hinge entirely on some digital engineering process $[2,3,6]$ on blocks, which leads to dividing them into hit and non-hit elements. We start with the 2-variable case [3, Lem. 3.3].

Lemma 2.7. Let $G$ be a block in $\mathrm{L}(2)$ of degree $2^{s}-1$. Then $G$ is equivalent to each of the following equivalent blocks $G_{i}, G_{i}^{\prime}$.

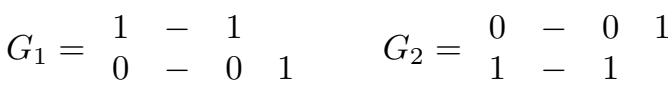

$$
\begin{aligned}
& G_{1}^{\prime}=\begin{array}{lll}
1 \\
0 & 1 & -1
\end{array} \quad G_{2}^{\prime}=\begin{array}{lll}
0 & 1 & -1 \\
1 &
\end{array}
\end{aligned}
$$

We now turn our attention to three variables and continue in three cases.

\subsection{Case $\mu(d)=2, d=2\left(2^{t}-1\right)$}

Under these conditions the $\omega$-vector of a minimal spike is $(2, \ldots, 2)$, with length $t$. By Lemma 2.6 there is no higher $\omega$-vector and any monomial of lower $\omega$-order is hit [3, Lem. 4.1].

Lemma 2.8. Let $F$ be a block in $\mathrm{L}^{d}(3)$, where $d=2^{t+1}-2$, with $t>1$. Then $F$ is non-hit if and only if $\omega(F)=(2, \ldots, 2)$. In this case $F$ is equivalent to one of the following types of block.

$$
\begin{aligned}
& F_{1}=\begin{array}{lll}
1 & - & 1 \\
1 & - & 1
\end{array} \quad F_{2}=\begin{array}{lllll}
1 & - & 1 & \\
1 & & 1 & 1
\end{array} \\
& 0-0 \quad 1 \quad 0 \quad-01 \\
& 1-1 \quad 1-11 \\
& F_{3}=\begin{array}{lllllllllll}
0 & - & 0 & 1
\end{array} \quad F_{4}=\begin{array}{lllll}
1 & - & 0 & 1
\end{array}
\end{aligned}
$$

More precisely, $F$ is equivalent to $F_{i}$, for $1 \leq i \leq 3$, if the $\alpha$-count of row $i$ is equal to $t$, and $F$ is equivalent to type $F_{4}$ if the $\alpha$-count of every row of $F$ 
is less than $t$. In each case, the hit equation has the form $F \cong F_{i}+E$, where $\omega(E)=(2, \ldots, 2,0,1, \ldots, 1)$ in which the 1 -section exists but the 2 -section may be empty. Moreover, the $F_{i}$ are linearly independent in $\mathrm{Q}^{d}(\mathrm{P}(3))$.

\subsection{Case $\mu(d)=1$}

In this situation $d=2^{s}-1$ and by Lemma 2.6 the least $\omega$-vector in degree $d$ is $(1, \ldots, 1)$ of length $s$. We also know that any monomial $g$ of degree $d$ is non-hit (see [3, Theorem 2.6]). If $s=1,2$, then $g \in \mathrm{L}^{d}(3)$ is a spike. The next lemma [3, Lem. 4.2] gives more detailed information.

Lemma 2.9. Let $G$ be a non-spike block in $\mathrm{L}^{d}(3)$, where $d=2^{s}-1$, with $s>2$. Then $G$ is equivalent to a linear combination of the following blocks.

$$
\begin{aligned}
& \begin{array}{llllllllll}
1 & 1 & - & 1 & 1 & 1 & - & 1 & 1 & -1
\end{array} \\
& G_{1}=\begin{array}{llll}
1 & 1 & - & 1
\end{array} \quad G_{2}=\begin{array}{llllllllllll}
1 & 1 & - & 1 & 1
\end{array} \quad G_{3}=\begin{array}{llllll}
1 & 0 & - & 0 & 1
\end{array}
\end{aligned}
$$

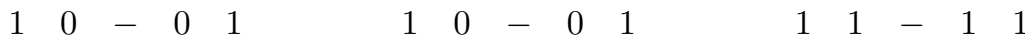

$$
\begin{aligned}
& 11-11-1
\end{aligned}
$$

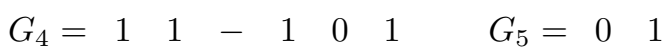

$$
\begin{aligned}
& 1 \quad 0 \quad-\quad 0 \quad 1 \quad 1 \quad 0001
\end{aligned}
$$

More precisely, if $\omega(G) \neq(3,2, \ldots, 2)$, then $G \cong G_{5}$ and if $\omega(G)=(3,2, \ldots, 2)$, then, either $G$ is a spike or, $G$ is equivalent to a linear combination of $G_{i}+G_{5}$ for $1 \leq i \leq 3$ if the $\alpha$-count of row $i$ is equal to $s-1$, and $G$ is equivalent to a linear combination of $G_{4}+G_{5}$ if the $\alpha$-count of every row of $G$ is less than $s-1$. These blocks are linearly independent in $\mathrm{Q}^{d}(\mathrm{P}(3))$.

2.3. Case $\mu(d)=2, d=2^{t+s}+2^{t}-2, t, s>0$

The next result here helps us to handle the standard splitting factors $[3$, Lem. 4.3].

Lemma 2.10. In the case $n=3, \mu(d)=2$, the factors $B^{t}, B^{s}$ of the standard splitting $B=B^{t} B^{s}$ can be manipulated independently in their equivalence classes without changing the equivalence class of $B$.

The following result summarizes the situation so far.

Lemma 2.11. Let $d=2^{t+s}+2^{t}-2$, with $t, s>0$, and let $B$ be a block in $\mathrm{L}^{d}(3)$, not lower than the minimal spike in degree $d$. Then, $B$ can be taken in the standard form $B^{t} B^{s}$ where $B^{t}$ is either a spike in two variables, or $B^{t} \cong F_{i}$ is one of the canonical forms in Lemma 2.8, and $B^{s}$ is either a spike in one or three variables (with $\omega$-vector $(3,2, \ldots, 2))$ or $B^{s} \cong G_{5}$, or $B^{s}$ is equivalent to a linear combination of $G_{j}+G_{5}$ for $1 \leq j \leq 4$, where $G_{j}$ is one of the canonical forms exhibited in Lemmas 2.7 or 2.9 .

In particular, if $B$ is not hit, then $B$ satisfies the property in Lemma 2.11. Is the converse of Lemma 2.11 true? That is, if a block $B$ satisfies the property in Lemma 2.11, is it non-hit? We answer this question in the following lemmas [3, Lems. 4.5 and 4.6]. 
Lemma 2.12. Let $d=2^{t+s}+2^{t}-2$, with $t, s>0$, and let $B$ be a block in $\mathrm{L}^{d}(3)$, not lower than the minimal spike in degree $d$. Suppose that $B=B^{t} B^{s}$, where $B^{t}$ is a 2-variable spike. Then $B$ is non-hit.

Lemma 2.13. With the notation as in Lemma 2.11, the block $B=F_{4} G$ with $G$ in the position of $B^{s}$ in Lemma 2.11 is hit if and only if every row of $G$ is nonzero.

\section{Proofs}

One way of demonstrating that a monomial $f$ in $\mathrm{P}(n)$ is not hit is to transform $f$ by a suitable element in the semigroup ring of $n \times n$ matrices into an expression which contains a spike. Since the spikes do not occur in any hit equation, and the matrix action commutes with the Steenrod action, it follows that $f$ is non-hit. A simple example of this procedure is to equate variables to each other, so called specialization in classical algebra. The specialization devices break down when $\sigma(g)$ has an even number of monomials in its orbit. We have to resort to an examination of all possible relations, which is a formidable task in general. However, in the 3 -variable case the $\omega$-vectors are sufficiently restricted to allow the analysis to be carried out in terms of the splicing technique $[2,3,6]$.

By Theorems 2.3 and 2.4 we can restrict attention to degrees for which $\mu(d)=1$ or 2 and we may work in $\mathrm{L}(3)$ since the 1 -variable and 2 -variable cases have been settled. As far as linear independence goes, spikes can be ignored since they cannot enter nontrivial relations.

\subsection{Case $\mu(d)=2$}

Let $d=2^{t+s}+2^{t}-2$ and $t>0$. The challenge here is to classify the symmetrized monomials into hit and non-hit classes.

Lemma 3.1. Let $F$ be a block in $\mathrm{L}^{d}(3)$ of degree $d=2^{t+1}+2^{t}-2$, with $t>0$. Then $F$ is non-hit if and only if $\omega(F)=(2, \ldots, 2,1)$. In this case $F$ is equivalent to one of the following types of blocks, up to permutation of rows.

$$
\begin{aligned}
& F_{1}^{\prime \prime}=\begin{array}{lllllllll}
1 & - & 1 \\
1 & - & 1 \\
0 & - & 0 & 1
\end{array} \quad F_{2}^{\prime \prime}=\begin{array}{lllll}
1 & - & 1 & 1 & 1 \\
1 & - & 1 & & \\
0 & - & 0 & 1
\end{array} \\
& F_{3}^{\prime \prime}=\begin{array}{llllll}
1 & - & 1 & 1 \\
1 & - & 1 & 0 & 1 \\
0 & - & 0 & 1
\end{array} \quad F_{4}^{\prime \prime}=\begin{array}{lllllll}
1 & - & 1 & 1 & 0 & 1 \\
1 & - & 1 & 0 & 1 & \\
0 & - & 0 & 1 & 1
\end{array}
\end{aligned}
$$

Proof. The first part of the proof follows the same line of argument as the first part of the proof of Lemma 2.8 in [3]. The second part follows from Lemma 2.11 . 
An element $\pi$ in $\Sigma_{n}$ acts on a monomial by permuting the variables. The notation $\pi_{(i, j)}$ is used for the element of $\Sigma_{n}$ which switches $x_{i}$ and $x_{j}$ and leaves the other variables fixed.

Proof of Theorem 1.1. Clearly $F$ is non-spike. By Theorem 2.5, if $F$ is below a minimal spike, then $\sigma(F)$ is symmetrically hit. Otherwise, up to permutation of rows and up to equivalence, $F$ is equal to $F_{1}$ or $F_{4}$ in Lemma 2.8 when $s=0$, or is equivalent to one of $F_{1}^{\prime \prime}$ to $F_{4}^{\prime \prime}$ in Lemma 3.1 when $s=1$. For the block $F_{1}^{\prime \prime}$, splicing the 0 -section in row 3 shows that $\sigma\left(F_{1}^{\prime \prime}\right)$ is symmetrically hit. Now let $F$ be one of $F_{1}, F_{4}, F_{2}^{\prime \prime}, F_{4}^{\prime \prime}$. Then by splicing the 0 -section in row 3 of $F$ we obtain $F \cong F \pi_{(2,3)}$. Hence by Part 3 of Proposition $2.1, \sigma(F)$ is symmetrically hit. Finally, one-back splicing the zero in row 2 of $F_{3}^{\prime \prime}$ gives $F_{3}^{\prime \prime} \cong F_{3}^{\prime \prime} \pi_{(1,2)}+F_{1}^{\prime \prime}$. Summing over cyclic permutations of $1,2,3$ and using the fact that $\sigma\left(F_{1}^{\prime \prime}\right)$ is symmetrically hit, shows that $\sigma\left(F_{3}^{\prime \prime}\right)$ is symmetrically hit.

In $[6$, Thm. 1.2] we proved that the symmetrized spikes together with the symmetrizations of canonical monomials $\mathfrak{c}=x_{1}^{d_{1}} x_{2}^{d_{2}} x_{3}^{d_{3}}$ of three types

$$
\begin{array}{lll}
\mathfrak{c}_{1}: d_{1}=2^{u}+2^{u+t+s-1}-1, & d_{2}=2^{u+t+s-1}-1, d_{3}=2^{u+t}-1 & s>1, t>0 \\
\mathfrak{c}_{2}: d_{1}=2^{u+2}-1, & d_{2}=d_{3}=2^{u+s-1}-2^{u}-1 & s>3 \\
\mathfrak{c}_{3}: d_{1}=2^{u+s-1}-1, & d_{2}=d_{3}=2^{u}+2^{u+s-2}-1 & s>2,
\end{array}
$$

where $u \geq 0$ in all cases, generate $\mathrm{B}(3)$ over the Steenrod algebra.

Proof of Theorem 1.2. Since $\mu(d)=2$, in the $(u, t, s)$-convention we have $u=0$ and the standard splitting $\mathfrak{M}=\mathfrak{M}^{t} \mathfrak{M}^{s}$. Therefore, any block $G$ splits $G=$ $G^{t} G^{s}$ and the canonical monomial $\mathfrak{c}_{1}$ splits

$$
\mathfrak{C}_{1}=\begin{array}{lllllll}
0 & - & 0 & 0 & - & 0 & 1 \\
1 & - & 1 & 1 & - & 1 & \\
1 & - & 1 & & & &
\end{array}
$$

The next definition partitions the set of blocks in $\mathrm{L}^{d}(3)$ into two subsets which will ultimately describe the symmetrically hit and non-hit symmetrized blocks.

Definition 3.2. Let $d=2^{t+s}+2^{t}-2$, with $t>0$. Let $\mathcal{V}$ denote the set of blocks $V \in \mathrm{L}^{d}(3)$ satisfying either of the following two conditions.

(a) $\omega(V)=\omega(\mathfrak{M})$ and, in addition, in the standard splitting $V=V^{t} V^{s}$, the block $V^{s}$ has exactly one zero row.

(b) $V$ has one of the following forms up to permutation of rows.

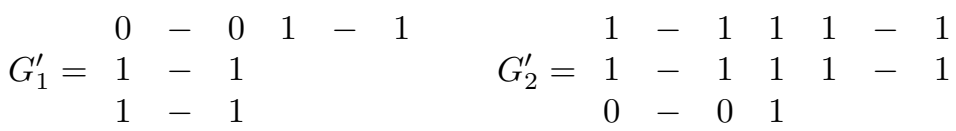

Let $\mathcal{H}$ be the complementary set of blocks of $\mathrm{L}^{d}(3)$. 
To simplify the wording of the next statement and proof we shall use $V$, with possible adornments, for a typical element of $\mathcal{V}$ and similarly $H$ for an element of $\mathcal{H}$.

Proposition 3.3. Let $d=2^{t+s}+2^{t}-2$ with $t>0$ and $s>1$ and let $F \in$ $\mathrm{P}^{d-2^{k}}(3)$. If the expansion of $S q^{2^{k}}(\sigma(F))$ contains an element $\sigma(V)$ as a term, then modulo elements $\sigma(H), S q^{2^{k}}(\sigma(F))$ contains exactly one other element $\sigma\left(V^{\prime}\right)$, where $V^{\prime}$ is not any permutation of $V$.

Proof. First we note that $F$ is a splicing of $V$. If $V$ is $G_{1}^{\prime}$ or $G_{2}^{\prime}$, it is easily proved that, modulo elements $\sigma(H)$,

$$
S q^{2^{k}}(\sigma(F)) \cong \sigma(V)+\sigma\left(V^{\prime}\right),
$$

where $V^{\prime}$ satisfies the condition (a) in Definition 3.2. So, we assume $V$ is of type (a) in Definition 3.2 and consider its splicing from the start position $i$ to the end position $j$ in two cases.

If $t<i$ or $j<t$, we have a hit equation as (1), where $V^{\prime}$ is of type (a) in Definition 3.2, different from any permutation of $V$. Assume, therefore, $i \leq t \leq j$. In this case we obtain the same hit equation (1) but, this time, $V^{\prime}$ is either one of $G_{1}^{\prime}, G_{2}^{\prime}$ or an element of type (a) in Definition 3.2, different from any permutation of $V$. This completes the proof.

The next result partitions the symmetrization of the monomials in the case $\mu(d)=2$ into hit and non-hit classes.

Lemma 3.4. (1) If $V \in \mathcal{V}$, then $\sigma(V) \cong \sigma\left(\mathfrak{C}_{1}\right)$;

(2) If $H \in \mathcal{H}$, then $\sigma(H)$ is symmetrically hit;

(3) For any block $G \in \mathrm{L}^{d}(3), \sigma(G)$ is not symmetrically hit if and only if $G \in \mathcal{V}$.

Proof. The proof goes through a number of intermediate steps, starting with some specific equivalence relations involving the blocks $G_{1}^{\prime}, G_{2}^{\prime}$ in Definition 3.2 and the following blocks.

$$
G_{1}^{\prime \prime}=\begin{array}{lllllll}
1 & - & 1 & 1 \\
1 & - & 1 & 0 & 1 \\
0 & - & 0 & 1
\end{array}
$$

Splicing the 0-section of $G_{2}^{\prime}$ and applying Corollary 2.2 gives $\sigma\left(G_{2}^{\prime}\right) \cong \sigma\left(\mathfrak{C}_{1}\right)$. By Lemma 2.10, $\sigma\left(G_{2}^{\prime \prime}\right) \cong \sigma\left(\mathfrak{C}_{1}\right)$. Now, splicing the 0 -section of $G_{2}^{\prime \prime}$ leads to $\sigma\left(G_{1}^{\prime}\right) \cong \sigma\left(\mathfrak{C}_{1}\right)$. Finally, one-back splicing the zero at position $(2, t)$ of $G_{1}^{\prime \prime}$ shows by Part 1 of Proposition 2.1 that $\sigma\left(G_{1}^{\prime \prime}\right) \cong 0$.

We now assume that $V$ satisfies the condition (a) of Definition 3.2. By Lemma 2.10 we may also assume, up to equivalence, that $V^{s}$ has one of the 2variable canonical forms exhibited in Lemma 2.7, up to row-permutation. Any such equivalence of $V$ symmetrizes by Part 1 of Proposition 2.1 since the rows of $V$ are distinct. Then, either $V$ has the form $G_{2}^{\prime \prime}$, up to row-permutation, or 
the rows of $V^{t}$ are all nonzero. Another application of Lemma 2.10 reduces the problem to the case where $V$ is one of the following types of blocks.

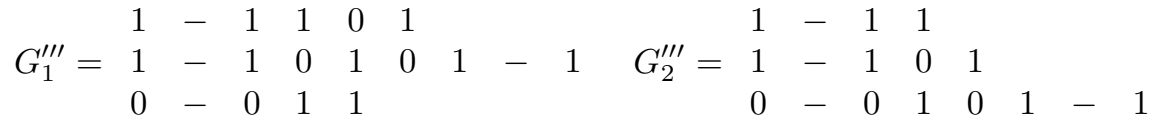

$$
\begin{aligned}
& G_{3}^{\prime \prime \prime}=\begin{array}{llllllll}
1 & - & 1 & 1 & 1 & - & 1 & \\
1 & - & 1 & 0 & 0 & - & 0 & 1 \\
0 & - & 0 & 1 & & & &
\end{array}
\end{aligned}
$$

One-back splicing at position $(1, t)$ in $G_{1}^{\prime \prime \prime}$ and appealing to Lemma 2.9 gives $\sigma\left(G_{1}^{\prime \prime \prime}\right) \cong \sigma\left(G_{2}^{\prime \prime \prime}\right)$. By one-back splicing at position $(2, t)$ in $G_{2}^{\prime \prime \prime}$ and using Corollary 2.2 we get $\sigma\left(G_{2}^{\prime \prime \prime}\right) \cong \sigma\left(G_{1}^{\prime}\right)$. Finally, by splicing the 0-section in row 2 of $G_{3}^{\prime \prime \prime}$ we have $\sigma\left(G_{3}^{\prime \prime \prime}\right) \cong \sigma\left(G_{2}^{\prime}\right)$. Hence in all cases $\sigma(V) \cong \sigma\left(\mathfrak{C}_{1}\right)$ for $V \in \mathcal{V}$, as required in Part 1.

For Part 2, we may assume that $H$ is not lower than the minimal spike $\mathfrak{M}$ in which case $\omega\left(H^{t}\right)=\omega\left(\mathfrak{M}^{t}\right)$ and $\operatorname{deg}\left(H^{s}\right)=2^{s}-1$. There are two preliminary cases to deal with.

If $H^{s}$ has exactly one nonzero row, then by Lemmas 2.8 and 2.10 , the problem reduces to an examination of the following types of blocks.

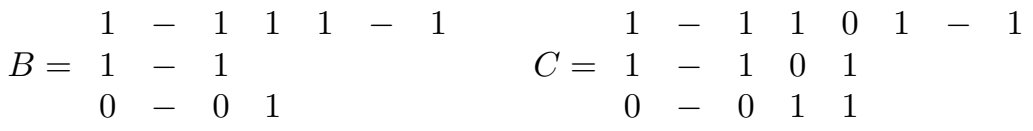

By splicing the 0 -section in row 3 of each block $B, C$ and applying Part 3 of Proposition 2.1 we see that both blocks are symmetrically hit.

It remains to verify the case where $H^{s}$ has three nonzero rows. By Lemma $2.11, H^{t}$ is either a 2-row spike or, up to row-permutation and up to equivalence, is one of the canonical forms $F_{1}, F_{4}$ in Lemma 2.8. If $H^{t}=F_{4}$, then Lemma 2.13 says that $H$ is hit and hence $\sigma(H)$ is symmetrically hit. We now continue the argument in two subcases.

If $\omega\left(H^{s}\right) \neq(3,2, \ldots, 2)$, then by Lemma 2.9 we may assume $H^{s}=G_{5}$, the canonical form in that lemma. Therefore, up to permutation of rows, $H$ is equivalent to one of the following blocks.

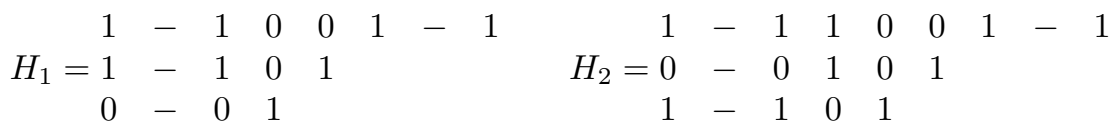

An application of Part 1 of Proposition 2.1 for one-back splicing at position $(3, t)$ in each block gives $\sigma\left(H_{1}\right) \cong 0 \cong \sigma\left(H_{2}\right)$.

On the other hand, if $\omega\left(H^{s}\right)=(3,2, \ldots, 2)$, then we may assume $H^{s}$ is one of the canonical forms $G_{i}$ in Lemma 2.9 for $1 \leq i \leq 4$. Now, one-back splicing of the single zero in column $t$ of $H$ produces the sum of two blocks with $\omega$-vectors satisfying the previous subcase. This settles Part 2. 
We now prove Part 3. Proposition 3.3 says that for $V \in \mathcal{V}$ in any relation involving $\sigma(V)$, there must always be an even number of terms of this kind. It follows that $\sigma(V)$ is not symmetrically hit. This proves Part 3 and completes the proof of Lemma 3.4 .

The proof of Theorem 1.2 is now completed as it follows immediately from Lemma 3.4.

\subsection{Case $\mu(d)=1$}

Proof of Theorem 1.3. The procedure of the proof follows the same lines of development as in Definition 3.2 and Lemma 3.4. To achieve this, we need some preliminary results.

Recalling the $\alpha, \alpha^{*}$-vectors in the introductory section, we partition nonspike blocks in $\mathrm{L}^{d}(3)$, having at least two equal rows into two sets

$$
\begin{aligned}
& \mathcal{Y}=\left\{Y \in \mathrm{L}^{d}(3): \alpha^{*}(Y)=(2, s-2, s-2)\right\}, \\
& \mathcal{Z}=\left\{Z \in \mathrm{L}^{d}(3): \alpha^{*}(Z)=(i, i, s-i), 0<i<s-2\right\} .
\end{aligned}
$$

The other non-spike blocks, which have distinct rows, are partitioned into two sets

$$
\begin{aligned}
& \mathcal{V}=\left\{V \in \mathrm{L}^{d}(3): \omega(V)=(3,2, \ldots, 2)\right\}, \\
& \mathcal{H}=\left\{H \in \mathrm{L}^{d}(3): \omega(H) \neq(3,2, \ldots, 2)\right\} .
\end{aligned}
$$

For convenience, letters $Y, Z, V, H$, possibly with embellishments, will denote elements in the sets $\mathcal{Y}, Z, V, H$, respectively. The analogue of Proposition 3.3 and Lemma 3.4 goes as follows.

Proposition 3.5. Let $\mathcal{B}$ denote one of the sets $\mathcal{Y}, \mathcal{Z}$, or $\mathcal{V}$ and $B \in \mathcal{B}$. Let $P \in \mathrm{P}^{d-2^{k}}(3)$, where $d=2^{s}-1$ with $s>3$ and assume that the expansion of $S q^{2^{k}}(\sigma(P))$ contains $\sigma(B)$ as a term. Then, only one of the following relations holds modulo elements $\sigma(H)$.

$$
\begin{aligned}
& S q^{2^{k}}(\sigma(P)) \cong \sigma(B)+\sigma\left(B^{\prime}\right), \\
& S q^{2^{k}}(\sigma(P)) \cong \sigma(Y)+\sigma(Z)+\sigma(V),
\end{aligned}
$$

where in $(2) B^{\prime}$ is not any permutation of $B$ and both $B, B^{\prime}$ belong to exactly one of $\mathcal{Y}, \mathcal{Z}$, or $\mathcal{V}$, and in (3) one of $Y, Z$, or $V$ is $B$.

Proof. It is clear that $P$ is a splicing of $B$. We consider all possible splicings. The expansion of $S q^{2^{k}}(P)$ contains an element of each of the sets $\mathcal{Y}, \mathcal{Z}, \mathcal{V}$ if and only if, up to row-permutation, $P$ has the form

$$
P=\begin{array}{lllllllllllll}
1 & 0 & - & 0 & 0 & 1 & - & 1 & 1 & & & \\
1 & 1 & - & 1 & 1 & 1 & - & 1 & 0 & 1 & - & 1 \\
1 & 1 & - & 1 & 1 & 1 & - & 1 & 0 & 1 & - & 1
\end{array}
$$


where the middle section with $\omega$-vector $(3, \ldots, 3)$ is started from position $k+1$. Each of the sections with $\omega$-vectors $(2, \ldots, 2)$ may be empty. More precisely,

$$
S q^{2^{k}}(P)=Y+Z+V+V \pi_{(2,3)}+E,
$$

where all blocks in the error term $E$ are in $\mathcal{H}$. Symmetrizing the above hit equation gives the relation (3).

Suppose that the splicing $P$ of $B$ has a form different from the sample (4). Assume first that $P$ has distinct rows. Then we have

$$
S q^{2^{k}}(P)=B+B^{\prime}+\sum_{i} H_{i}
$$

where $B, B^{\prime}$ is indeed in $\mathcal{V}$. We see that $B^{\prime}$ is not any permutation of $B$ since $P$ is supposed to have distinct rows. Symmetrizing (5) now leads to the relation (2).

The problem is now reduced to the case where $P$ is not of the form (4) with at least two equal rows, say, 2,3. If the $\alpha$-counts of rows 2,3 of $P$ are $s-2$, then $P$ must have the form

$$
P=\begin{array}{rrrrrrrrr}
1 & 0 & - & 0 & 1 & & & \\
1 & 1 & - & 1 & 0 & 1 & - & 1 \\
1 & 1 & - & 1 & 0 & 1 & - & 1
\end{array}
$$

where the last digit 1 in the first row is situated at position $k+1$. The hit equation in this case is also of the form (5) where, here, $B, B^{\prime}$ are two different elements of $\mathcal{Y}$ since the action of $S q^{2^{k}}$ preserves the $\alpha$-counts.

On the other hand, if the $\alpha$-counts of rows 2,3 of $P$ is less than $s-2$, then the same happens for $B, B^{\prime}$. Therefore, $B, B^{\prime}$ are now two different elements of $\mathcal{Z}$. This completes the proof of Proposition 3.5.

The next result shows that the only hit class in the case $\mu(d)=1$ is the class of symmetrizations of the monomilas in $\mathcal{H}$.

Lemma 3.6. (1) If $H \in \mathcal{H}$, then $\sigma(H)$ is symmetrically hit;

(2) If $Y \in \mathcal{Y}$, then $\sigma(Y) \cong \sigma\left(\mathfrak{C}_{2}\right)$;

(3) If $Z \in \mathcal{Z}$, then $\sigma(Z) \cong \sigma\left(\mathfrak{C}_{3}\right)$;

(4) If $V \in \mathcal{V}$, then $\sigma(V) \cong \sigma\left(\mathfrak{C}_{2}\right)+\sigma\left(\mathfrak{C}_{3}\right)$.

Proof. Given $H \in \mathcal{H}$, by Lemma 2.9, we have $H \cong G_{5}$, one of the canonical forms in that lemma. One-back splicing in row 2 of $G_{5}$ gives the equivalence $G_{5} \cong G_{5} \pi_{(1,2)}$ which shows that $\sigma\left(G_{5}\right)$ is symmetrically hit. This proves Part 1 .

To prove Part 2, we observe that every block of $\mathcal{Y}$ is a row-permutations of one of the blocks

$$
\begin{aligned}
& \begin{array}{llllllll}
1 & 1 & 1 & 0 & - & 0 & 0 & 1
\end{array}
\end{aligned}
$$

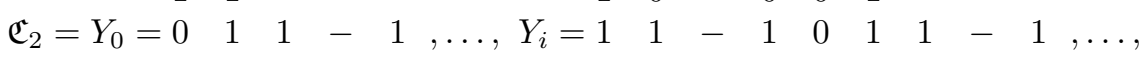

$$
\begin{aligned}
& \begin{array}{llllllllllllll}
0 & 1 & 1 & - & 1 & 1 & 1 & - & 1 & 0 & 1 & 1 & - & 1
\end{array}
\end{aligned}
$$




$$
Y_{s-2}=\begin{array}{llllll}
1 & 0 & - & 0 & 0 & 1 \\
1 & 1 & - & 1 & & \\
1 & 1 & - & 1 & &
\end{array}
$$

Simultaneous one-back splicing the zeros in the second and third rows of $Y_{i}$ gives the relation $Y_{i} \cong Y_{i+1}$ and establishes Part 2 .

For Part 3, we may assume the second and third rows as equal rows in the blocks with at least two equal rows. Let $0<i<s-2$ and denote by $\mathcal{Z}_{i}$ the subset of $\mathcal{Z}$ containing all blocks with $\alpha$-vectors $(s-i, i, i)$. Then

$$
\mathcal{Z}=\bigcup_{i=1}^{s-3} \mathcal{Z}_{i}
$$

Notice that $\mathfrak{C}_{3} \in \mathcal{Z}_{1}$. Figure 1 , in the case $s=6$, illustrates all blocks in three sets $\mathcal{Z}_{1}, \mathcal{Z}_{2}, \mathcal{Z}_{3}$, separated by dots.

For $1 \leq i \leq s-3$ in any $\mathcal{Z}_{i}$, there is only one block $Z_{i}^{\prime}$, up to row-permutation, in which the first row has no 0 followed by any 1 . In Figure 1, these blocks are displayed in bold fonts. Take an arbitrary block $Z_{i} \in \mathcal{Z}_{i}$, different from $Z_{i}^{\prime}$. Then by iterative one-back splicing in the first rows, we obtain a finite chain of equivalences modulo $\mathcal{H}$ which shows $Z_{i} \cong Z_{i}^{\prime}$. This implies $\sigma\left(Z_{i}\right) \cong \sigma\left(Z_{i}^{\prime}\right)$. In Figure 1, the direction of the achievement of the above argument is denoted by single arrows.

To join the equivalence classes of the $\sigma\left(Z_{i}^{\prime}\right)$ together and achieve a one-piece equivalence class, let $1<i \leq s-3$ and consider the greatest, in $\omega$-order, block

$$
\begin{array}{llllllll}
1 & 0 & - & 0 & 0 & 1 & - & 1 \\
1 & 1 & - & 1 & & & & \\
1 & 1 & - & 1 & & & &
\end{array}
$$

in $\mathcal{Z}_{i}$. Splicing the 0 -section of the above block produces, modulo $\mathcal{H}$, a block with $\alpha$-vector $(s-1,1,1)$ in $\mathcal{Z}_{1}$. This proves Part 3 . In Figure 1 , the symbol $\cong$ stands for these connections.

To prove the final part of the lemma, define $\mathcal{V}_{1}$ to be the set of all blocks of $\mathcal{V}$ having a row with $\alpha$-count equal to $s-1$, and $\mathcal{V}_{2}$ the set of all blocks of $\mathcal{V}$ whose rows have $\alpha$-count less than $s-1$. Then, by Lemma 2.9, up to permutation of rows and modulo $\mathcal{H}$, each element of $\mathcal{V}_{1}$ is equivalent to $G_{1}$ and each element of $\mathcal{V}_{2}$ is equivalent to $G_{4}$, the canonical forms in that lemma. Splicing the 0 -sections in row 3 of each of $G_{1}, G_{4}$ we get

$$
\sigma\left(G_{1}\right) \cong \sigma\left(\mathfrak{C}_{2}\right)+\sigma\left(\mathfrak{C}_{3}\right) \cong \sigma\left(G_{4}\right) .
$$

This proves Part 4 and completes the proof of Lemma 3.6.

We need the linear independence of $\sigma\left(\mathfrak{C}_{2}\right)$ and $\sigma\left(\mathfrak{C}_{3}\right)$.

Lemma 3.7. $\sigma\left(\mathfrak{C}_{2}\right), \sigma\left(\mathfrak{C}_{3}\right)$ are linearly independent.

Proof. By specialization of the variables to a single variable it is easily proved that $\sigma\left(\mathfrak{C}_{2}\right), \sigma\left(\mathfrak{C}_{3}\right)$ are not symmetrically hit, since each orbit has three elements. On the other hand, from Propositions 3.5 and 3.6 it follows that any 


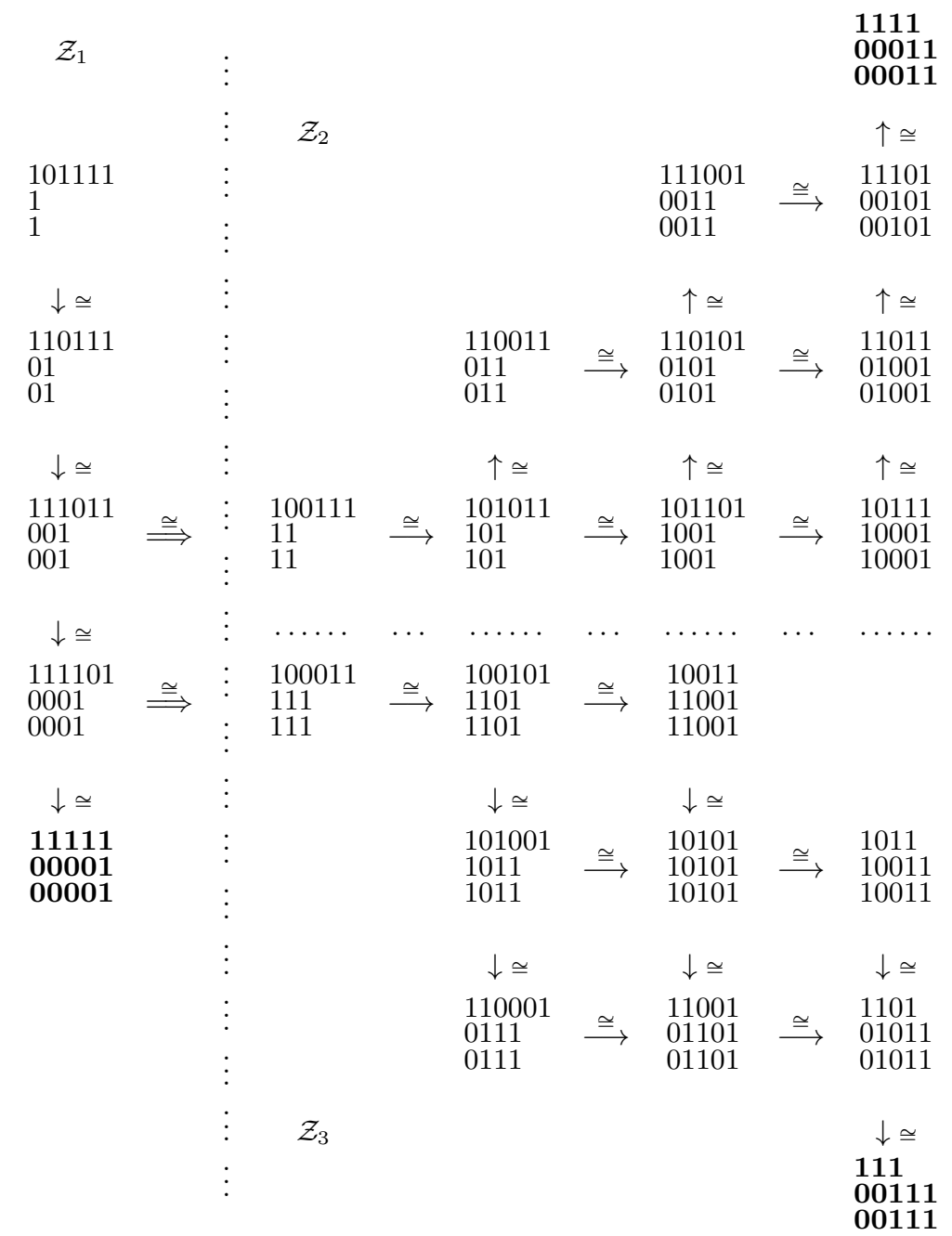

FiguRE 1. Subsets $\mathcal{Z}_{i}(i=1,2,3)$ of $\mathcal{Z}$ containing blocks with $\alpha$-vector $(s-i, i, i)$, for $s=6$

hit equation contains an even number of elements of the form $\sigma(Y)$ and an even number of elements of the form $\sigma(Z)$, where $Y \in \mathcal{Y}, Z \in \mathcal{Z}$. This completes the proof.

The proof of Theorem 1.3, now, follows from Lemmas 3.6 and 3.7. 


\section{Remarks}

In this section we state some open problems. Two basic aspects of the hit problem are to find a minimal generating set for familiar $\mathcal{A}_{2}$-modules and then to find criteria for their elements to be hit. In [4] we exhibited an important conjecture on the symmetric hit problem, posed in $[5,6]$.

Conjecture 4.1. For any monomial $f \in \mathrm{P}(n), n>0$, if $f$ is hit, then $\sigma(f)$ is symmetrically hit.

In Proposition 8.3 of [6] we proved Conjecture 4.1 for $n \leq 3$. On the other hand, Part 2 of Proposition 2.1 shows that Conjecture 4.1 is true for any $n$ in the case of distinct exponents. The converse of Conjecture 4.1, however, is not true as the following counterexample shows.

Example 1. Let $F$ be the following block.

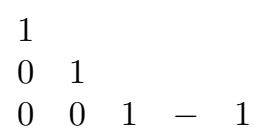

Then it can be easily checked that all permutations of $F$ are equivalent to each other and hence $\sigma(F)$ is symmetrically hit, while it is clear from [3, Lemma $2.6]$ that $F$ is non-hit.

For a graded left $\mathcal{A}_{2}$-module $\mathrm{M}$, we denote by $\mathrm{Q}(\mathrm{M})$ the quotient of the module $\mathrm{M}$ by the hit elements. Then $\mathrm{Q}(\mathrm{M})$ is a graded vector space over $\mathbb{F}_{2}$ and a basis for $\mathrm{Q}(\mathrm{M})$ lifts to a minimal generating set for $\mathrm{M}$ as a module over $\mathcal{A}_{2}$. Kameko [7] computed $\operatorname{dim}\left(\mathrm{Q}^{d}(\mathrm{P}(n))\right)$ for $n=1,2,3$ and conjectured a best upper bound $\prod_{k=1}^{n}\left(2^{k}-1\right)$ for general $n$. Nguyen Sum [10] showed that this conjecture is true for $n=4$. However, in a recent paper [11] he proved that this conjecture turns out to be wrong for any $n>4$. Therefore, a challenging problem is to find the best upper bound for $\operatorname{dim}\left(\mathrm{Q}^{d}(\mathrm{P}(n))\right)$.

On the other hand, we showed [6] that the best upper bound for the dimension of $\mathrm{Q}^{d}(\mathrm{~B}(n))$ in cases $n=1,2,3$ are respectively $1,1,4$. Therefore, finding the best upper bound for $\operatorname{dim}\left(\mathrm{Q}^{d}(\mathrm{~B}(n))\right)$ is also of importance.

\section{References}

[1] D. P. Carlisle and R. M. W. Wood, The boundedness conjecture for the action of the Steenrod algebra on polynomials, Adams Memorial Symposium on Algebraic Topology, 2 (Manchester, 1990), 203-216, London Math. Soc. Lecture Note Ser., 176, Cambridge Univ. Press, Cambridge, 1992.

[2] A. S. Janfada, The Hit Problem for Symmetric Polynomials Over the Steenrod Algebra, PhD thesis, Manchester University, UK, 2000.

[3] _ A criterion for a monomial in $\mathrm{P}(3)$ to be hit, Math. Proc. Cambridge Philos. Soc. 145 (2008), no. 3, 587-599.

[4] (2011), no. 3, 403-408.

[5] A. S. Janfada and R. M. W. Wood, The hit problem for symmetric polynomials over the Steenrod algebra, Math. Proc. Cambridge Philos. Soc. 133 (2002), no. 2, 295-303. 
[6] Generating $\mathrm{H}^{*}\left(B O(3), F_{2}\right)$ as a module over the Steenrod algebra, Math. Proc. Cambridge Philos. Soc. 134 (2003), no. 2, 239-258.

[7] M. Kameko, Generators of the cohomology of $B V_{3}$, J. Math. Kyoto Univ. 38 (1998), no. 3, 587-593.

[8] F. P. Peterson, A-generators for certain polynomial algebras, Math. Proc. Cambridge Philos. Soc. 105 (1989), no. 2, 311-312.

[9] L. Smith, Polynomial Invariants of Finite Groups, Res. Notes Math., vol. 6, A. K. Peters Ltd. Wellesley, MA, 1995.

[10] N. Sum, The hit problem for the polynomial algebra of four variables, Seoul, November 3, (2007), http://www.kms.or.kr/data/asia/files/nguyensum.pdf

[11] , The negative answer to Kameko's conjecture on the hit problem, Adv. Math. 225 (2010), no. 5, 2365-2390.

Department of Mathematics

URMIA UNIVERSITY

P.O.Box 165, URMIA-IRAN

E-mail address: a.sjanfada@urmia.ac.ir; asjanfada@gmail.com 